\title{
Dificuldades enfrentadas por docentes do ensino superior frente ao contexto da pandemia de COVID-19
}

\author{
Jennifer Thalita Targino dos Santos ${ }^{1}$ \\ Alan Vinicius Assunção-Luiz ${ }^{1}$ (D) \\ Alison Luis Eburneo Pereira ${ }^{1}$ (D) \\ Anderson Ricardo Malmonge Barbosa Luciano ${ }^{2}$ (D) \\ Idinei Francisco Pires de Carvalho Filho ${ }^{2}$ (D) \\ Milena Jorge Simões Flória Lima Santos ${ }^{1}$ \\ Mônica Cristina Garbin ${ }^{3}$ (D) \\ ${ }^{1}$ Universidade de São Paulo (USP), Brasil; ${ }^{2}$ Universidade Estadual Paulista "Júlio de Mesquita Filho" (UNESP), \\ Brasil; ${ }^{3}$ Universidade Virtual do Estado de São Paulo (UNIVESP), Brasil.
}

Resumo. A pandemia do COVID-19 modificou o cronograma de atividades propostas para o ano letivo de 2020-21, a partir da substituição das aulas presenciais pelo ensino remoto emergencial (ERE). Para os professores do ensino superior, embora as boas práticas de ensino sejam uma preocupação constante das universidades, surgiram desafios diante do modo repentino de transição e implementação do ERE. Nesse sentido, este estudo objetivou investigar as principais dificuldades encontradas por docentes do ensino superior no Brasil, frente ao ERE durante a pandemia de COVID-19. A coleta de dados ocorreu entre dezembro de 2020 e fevereiro de 2021, utilizando-se um questionário eletrônico constituído por 19 questões objetivas. Participaram do estudo 234 docentes. Deste total, 77,4\% dos participantes apresentaram dificuldades durante o processo de adaptação para o ERE, sendo estas relacionadas a falta de capacitação ou de recursos tecnológicos por parte das instituições; problemas com o uso de tecnologias digitais (ferramentas e softwares); influência de dificuldades psicológicas; dificuldades associadas à burocracia administrativa ou com Segurança de Dados. Elucida-se que a transição deve ser realizada com planejamento e investimento na formação adequada dos professores, para que a educação em contexto virtual alcance o mesmo sucesso dos encontros presenciais. Palavras-chave: dificuldades pedagógicas; ensino remoto emergencial; pandemia; COVID-19; metodologias pedagógicas; tecnologias digitais.

\section{Dificultades para los profesores de enseñanza superior en el contexto de la pandemia de COVID-19}

Resumen. La pandemia de COVID-19 modificó el cronograma de actividades propuestas para el curso académico 2020-21 con la sustitución de las clases presenciales por la enseñanza remota de emergencia (ERE). Aunque las buenas prácticas docentes son una preocupación constante de las universidades, los profesores de enseñanza superior se enfrentaron a nuevos desafíos ante el carácter repentino de la transición y aplicación de la ERE. En este sentido, el objetivo del presente estudio fue investigar las principales dificultades que encontraron los profesores de enseñanza superior en Brasil en relación con la ERE durante la pandemia de COVID-19. Los datos se recogieron entre diciembre de 2020 y febrero de 2021, a través de un cuestionario electrónico compuesto por 19 preguntas objetivas. En el estudio participaron un total de 234 profesores. De este total, el 77,4\% de los participantes presentó dificultades durante el proceso de adaptación a la ERE, relacionadas con la falta de formación o de recursos tecnológicos por parte de las instituciones, problemas con el uso de tecnologías digitales (herramientas y software), influencia de las dificultades psicológicas, dificultades asociadas a la burocracia administrativa o a la seguridad de datos. Se señala que la transición debe llevarse a cabo con planificación e inversión en la formación adecuada para los profesores, para que la educación en un entorno virtual alcance el mismo éxito que la presencial.

Palabras clave: dificultades pedagógicas, enseñanza remota de emergencia, pandemia, COVID-19, metodologías pedagógicas, tecnologías digitales.

\section{Difficulties experienced by higher education teachers during the COVID-19 pandemic}

Abstract. The COVID-19 pandemic changed the schedule of activities proposed for the academic year 2020-21, in which face-to-face classes were replaced by emergency remote teaching (ERE). For higher education professors, although good teaching practices are a constant concern of universities, challenges have arisen due to the sudden transition and implementation of the ERE. For this reason, this study aimed to investigate the main difficulties encountered by higher education professors in Brazil, dealing with the ERE during the COVID-19 pandemic. Data collection took place between December 2020 and February 2021, using a electronic questionnaire consisting of 19 objective questions. 234 professors participated in the study, of which $77.4 \%$ of the participants had difficulties during 
the adaptation process for the ERE, and are related to the lack of training or technological resources given by the institutions; problems with the use of digital technologies (tools and software); influence of psychological difficulties; difficulties associated with administrative bureaucracy or with Data Security. It is clarified that the transition must be carried out with planning and investment in the adequate training of teachers, so that education in a virtual context achieves the same success as face-to-face meetings. Keywords: pedagogical difficulties; emergency remote learning; pandemics; COVID-19; pedagogical methodologies; digital technologies.

\section{Introdução}

Entre o final do ano de 2019 e o início de 2020, um novo vírus denominado "SARS-CoV-2" foi identificado como responsável por causar a doença COVID-19 (Zhou et al., 2020). Foi inicialmente, encontrado na China e, posteriormente, propagou-se ao redor do mundo, e em março de 2020, a Organização Mundial da Saúde (OMS) declarou que se tratava de uma pandemia. Inúmeros protocolos passaram a ser adotados visando conter a disseminação do vírus (WHO, 2020a). Entre eles, o isolamento social, que gerou impactos significativos na área da educação (Brasil, 2020; Jelińska \& Paradowski, 2021).

Naquele momento, o Ministério da Educação do Brasil por meio da portaria MEC $n^{0} 345$, de 19 de março de 2020, dispôs sobre a substituição das aulas presenciais, em caráter excepcional, para a utilização de meios e tecnologias de informação e comunicação, ou seja, pelo Ensino Remoto Emergencial (ERE), enquanto permanecesse a pandemia da COVID-19 (Brasil, 2020). As instituições de ensino superior (IES) suspenderam suas atividades presenciais, o que afetou, substancialmente, discentes e docentes (Brasil, 2020; Jelińska \& Paradowski, 2021). Algumas prioridades foram estabelecidas pelo governo, a fim de garantir a continuidade da educação no Brasil, incluindo o acesso ao material didático para os alunos (livros e apostilas digitais), processos de avaliações virtuais e preparo dos profissionais envolvidos (docentes e outros funcionários das instituições de ensino), sobre o uso das tecnologias digitais e ferramentas de comunicação (Daniel, 2020). Embora as boas práticas de ensino sejam uma preocupação constante das universidades, nem todas as instituições estavam preparadas para dar o suporte e treinamentos necessários para os docentes no ERE (Polydoro, 2020; Jelińska \& Paradowski, 2021).

Aqualidade e a eficácia educacional no ERE podem ser influenciadas pelas aulas exclusivamente online, impedindo o contato face-a-face, que é percebido tanto pelos alunos, quanto pelos docentes (Jelińska \& Paradowski, 2021). No entanto, espera-se que pela obtenção, compreensão e domínio dos diversos tipos de abordagens educacionais, tecnológicas e de instrumentos de ensino, o ERE possa suprir suficientemente as necessidades neste contexto (Daniel, 2020; Garbin \& Oliveira, 2021).

$\mathrm{O}$ uso de tecnologias associadas às metodologias de ensino adequadas pode inovar e flexibilizar os processos de aprendizagem, essenciais para o letramento digital (Suguimoto et al., 2017; Carneiro et al., 2019). Este conjunto de conhecimentos e habilidades permitem o uso de dispositivos eletrônicos e comandos via softwares, possibilitando a imersão de estudantes e professores no ensino e-learning (ensino eletrônico apoiado em tecnologias digitais) (Suguimoto et al., 2017; Silus et al., 2020). Ressalta-se que, antes de se trabalhar com a utilização de softwares e tecnologias 
digitais, é preciso instruir-se acerca das suas funcionalidades e as consequências de seu uso nas relações sociais, bem como as contribuições para o ensino-aprendizagem (Moore et al., 2021).

Diante do modo repentino de transição e implementação do ERE, que evidenciou a falta de treinamento e conhecimento necessário a essa prática, algumas dificuldades foram relatadas por parte dos docentes e alunos das instituições (Jelińska \& Paradowski, 2021). Alguns fatores que podem influenciar na qualidade do ensino e da aprendizagem, foram identificados: questões psicológicas, a ansiedade e frustração diante de tantas incertezas (Chaturvedi et al., 2021) e harmonia dos relacionamentos entre estudantes e professores (Brooks et al., 2020); dificuldades com o uso das tecnologias digitais de comunicação (TDIC's) (Silus et al., 2020; Dwivedi et al., 2020), exclusão digital (comum em países em desenvolvimento) e recursos tecnológicos inadequados (Arruda, 2020; Roggero, 2020); privacidade e segurança dos usuários (Jelińska \& Paradowski, 2021).

\subsection{A ação docente e o uso de tecnologias}

A formação de professores que atuam na educação básica no Brasil é realizada pelos cursos superiores de Licenciaturas ofertadas pelas IES. Para atuar no ensino superior basta um grau de mestre ou doutor, dependendo das exigências da instituição, de acordo com a Lei Educacional Brasileira 9.394, de 20 de dezembro de 1996 (Arroio et al., 2006), o que define a formação desses docentes são os cursos e o tipo de titulação recebida durante sua pós-graduação.

Neste contexto há outra lacuna que precisa ser explorada e que evidenciou-se durante a pandemia da Covid-19: o uso de tecnologias. O debate sobre a importância da formação de professores para o uso intencional de tecnologias não é novo no Brasil, mas tornou-se mais intenso nos últimos anos (Garbin \& Oliveira, 2021). Em dezembro de 2019, estabeleceram-se as Diretrizes Curriculares Nacionais para Professores da Educação Básica (Brasil, 2019). Foram definidas dez competências gerais necessárias ao docente em formação e há referências às tecnologias em apenas três delas. Assim como os alunos, os professores precisam ter habilidades digitais básicas para que haja uma contribuição efetiva para a educação de seus discentes (Sailer et al., 2021).

Tal discussão é importante, especialmente no cenário vivido durante os anos de 2020 e 2021, pois o tema é negligenciado nas formações de professores no contexto brasileiro. E o que se evidenciou neste processo foi que professores do ensino superior e básico combinaram seus saberes docentes e mergulharam no uso das tecnologias em diferentes contextos, para que a educação, de alguma forma, não parasse (Garbin \& Oliveira, 2021).

Maphalala e Adigun (2021) indicam que para a adoção do modelo de educação remota deve-se passar pelos temas: "planejamento; suporte técnico e treinamento para e-learning; Infraestrutura de Tecnologia da Informação e Comunicação e acessibilidade à Internet; aceitação do e-learning e utilização do Learning Management System; desenvolvimento de conteúdo para e-learning; e avaliação da eficácia do ensino por meio do e-learning". Para além disso, de acordo com Moore et al. (2021) e 
Jelińska e Paradowski (2021), a transição para o ERE depende de fatores que incluem comunicação, tecnologia e pedagogia, deste modo, é preciso ir além dos dispositivos tecnológicos, mas relacionar esses elementos.

Além dessas questões, dado o isolamento social, os docentes passaram a se preocupar com a segurança e bem-estar dos alunos e de seus familiares, sendo este considerado um fator estressante na atuação durante o ERE (Besser et al., 2020; Gelles et al., 2020). Como já apontado pela literatura atual, durante o exercício profissional e vida acadêmica os docentes e discentes podem enfrentar momentos ansiogênicos e estressantes que contribuem para complicações mentais e físicas (Costa \& Nebel, 2018; Assunção-Luiz et al., 2021). Esses fatores podem ter sido intensificados durante a pandemia, sendo relacionados às medidas restritivas e outros protocolos impostos para a contenção do coronavírus (Assunção-Luiz et al., 2021).

Nesse sentido, este estudo objetivou investigar as principais dificuldades encontradas por docentes de instituições brasileiras públicas e privadas, frente à modalidade de educação remota emergencial durante a pandemia de COVID-19.

\section{Metodologia}

\subsection{Casuística e delineamento do estudo}

Para atingirmos o objetivo proposto por esse estudo, foi utilizada uma abordagem quantitativa com desenho descritivo e transversal. A amostra foi constituída por docentes, de ambos os sexos e sem limitação de faixa-etária, de universidades públicas e privadas, procedentes de distintas regiões do Brasil, atuantes nos anos 2020 e 2021, que vivenciaram o contexto da pandemia da COVID-19. Foram excluídos aqueles que não responderam ao questionário dentro do período de coleta de dados estipulado pelos autores.

O presente estudo respeitou os princípios éticos preconizados pela Resolução 510/16 do Conselho Nacional de Saúde (Brasil, 2016). Antes de responder o instrumento de coleta de dados, os participantes obtiveram conhecimento acerca dos objetivos, riscos e benefícios do estudo, sendo assegurados sobre o sigilo de seus dados em todas as etapas. Posteriormente concederam sua anuência para a divulgação dos resultados da pesquisa em eventos e artigos científicos.

\subsection{Instrumento de coleta de dados}

Para atingir um número maior de docentes, o instrumento utilizado neste estudo foi um questionário eletrônico elaborado com base na literatura de referência (WHO, 2020b) e nas vivências e experiências dos autores, sendo esses um grupo multiprofissional com integrantes da área da educação e da saúde.

O referido questionário foi previamente testado por meio de um estudo piloto online, de outubro a novembro de 2020. Participaram deste estudo piloto 50 docentes do ensino superior, vinculados às mesmas instituições de ensino dos autores, que forneceram feedback acerca do questionário inicialmente proposto. Ressalta-se que esses participantes foram excluídos da amostragem final do estudo. Dentre as modificações realizadas, uma questão foi excluída e uma discursiva foi adaptada para objetiva. Além disso, foram sugeridos a inclusão de itens relacionados aos softwares 
utilizados pelos docentes: Google Jamboards, Jogos (exemplo: Kahoot, Socrative, Mentimeter etc) e bibliotecas virtuais (exemplo: Pearson, Minha Biblioteca etc). A redação dos enunciados foi mantida.

A versão final do questionário foi inserida na plataforma Google Forms, o que possibilitou que esta pesquisa pudesse ser respondida por diversos professores, em diferentes instituições brasileiras. Ademais, a coleta de dados realizada por meio eletrônico possibilitou que os participantes pudessem acessá-lo no momento e horário de sua maior conveniência. O questionário possuía um caráter auto-explicativo, possibilitando que orientações a respeito de sua utilização fossem verificadas pelos participantes durante o preenchimento dos dados, sem a necessidade de um cadastro prévio ou aplicativo instalado para o seu acesso.

As questões viabilizaram a investigação de fatores centrais relacionados ao objetivo desta pesquisa, tais como: dificuldades encontradas e a experiência prévia dos docentes na modalidade EaD; principais tecnologias utilizadas; e o uso de softwares. Foi composto por 19 perguntas de múltipla escolha, sendo dividido em duas partes. Compuseram a primeira parte nove questões referentes à caracterização da amostra (idade; sexo; procedência; área de formação; nivel de titulação; tempo de docência no ensino superior; número de instituições em que atua; caracterização das IES; área de atuação, segundo a classificação da Coordenação de Aperfeiçoamento Pessoal de Nivel Superior - CAPES) e a segunda parte foi composta por 10 questões a respeito do período de transição dos docentes para o ERE, sendo dividida em quatro categorias a saber: (1) Recursos tecnológicos e capacitação ofertados pelas instituições; (2) Dificuldades no uso de tecnologias digitais: Ferramentas e softwares utilizados durante o ensino remoto; (3) Dificuldades psicológicas e atuação do docente no ERE; (4) Aspectos burocráticos, avaliações pedagógicas e segurança de dados.

A coleta de dados ocorreu entre dezembro de 2020 e fevereiro de 2021. Os participantes foram convidados via e-mail e/ou por aplicativos de comunicação como o WhatsApp, Facebook, dentre outros. O convite incluía uma breve apresentação da pesquisa e o link de acesso ao formulário.

\subsection{Análise de dados}

Os dados coletados foram codificados, categorizados e editados para serem analisados usando estatística descritiva simples e a identificação de possíveis diferenças significativas entre grupos definidos.

A estatística descritiva simples foi obtida diretamente pela ferramenta do Google Forms, que fornece um relatório com todos os resultados mensurados, e permite caracterizar a amostra e as variáveis estudadas. Para identificar possíveis diferenças significativas entre grupos definidos, os dados foram analisados em ambiente estatístico R (4.0.1) e utilizou-se o teste exato de Fisher em comparação par-a-par. Valores de probabilidade $\alpha<0,05$ foram considerados estatisticamente significativos, juntamente com os valores de Odds Ratio (OR).

Sendo assim, avaliou-se as possíveis associações entre as dificuldades apresentadas pelos docentes e: (1) os recursos fornecidos pelas instituições de ensino; (2) os aspectos burocráticos (problemas com a direção, coordenação ou funcionários); 
(3) a avaliação discente; (4) as experiências prévias no ensino superior a distância (EaD); (5) as questões referentes à saúde mental; (6) os softwares utilizados no ERE; (7) a interatividade com os discentes.

\section{Resultados e discussão}

Levando-se em consideração o período de distanciamento social, a pesquisa realizada via Google Forms possibilitou atingir um número amostral suficiente $(n=234)$, conforme descrito por Silus et al. (2020), que sugerem um número mínimo de 200 participantes em pesquisas desse cunho. A caracterização dos participantes é apresentada na tabela 1 .

Tabela 1. Caracterização da amostra de acordo com: idade, sexo, tipo da instituição em que atua, tempo de atuação, número de instituições, maior nível de titulação, procedência, e áreas de atuação

\begin{tabular}{lr}
\hline Idade & $\begin{array}{r}\text { Média:46,45 } \\
\text { Moda: } 51 \\
\text { Mediana: } 46,5\end{array}$ \\
\hline Sexo & $\mathrm{N} \%)$ \\
Feminino & $111(47,4)$ \\
Masculino & $122(52,1)$ \\
Prefiro não informar & $1(0,4)$ \\
\hline Setor da instituição em que atua & $\mathrm{N}(\%)$ \\
Pública - Federal & $39(16,7)$ \\
Pública - Estadual & $158(67,5)$ \\
Pública - Municipal & $5(2,1)$ \\
Privada & $61(26,1)$ \\
\hline Anos de atuação & $\mathrm{N}(\%)$ \\
até 5 anos & $44(18,8)$ \\
de 6 a 10 anos & $44(18,8)$ \\
de 11 a 15 anos & $30(12,8)$ \\
de 16 a 20 anos & $39(16,7)$ \\
mais de 20 anos & $77(32,9)$ \\
\hline Quantidade de instituições que atua & $\mathrm{N}(\%)$ \\
1 instituição & $187(79,9)$ \\
2 instituições & $39(16,7)$ \\
3 instituições & $7(3,0)$ \\
4 ou mais instituições & $1(0,4)$ \\
\hline Maior nível de Titulação & $\mathrm{N}(\%)$ \\
Licenciatura & $1(0.4)$ \\
Bacharelado & $2(0.9)$ \\
Especialização & $3(1.3)$ \\
Mestrado & $38(16.2)$ \\
Doutorado & $90(38.5)$ \\
Pós-doutorado & $58(24.8)$ \\
\hline & $42(17.9)$ \\
\hline & \\
\hline
\end{tabular}




\begin{tabular}{lr}
\hline Estado de atuação no Brasil (\%) & N (\%) \\
São Paulo (SP) & $189(80,8)$ \\
Pernambuco (PE) & $23(9,8)$ \\
Rio de Janeiro (RJ) & $3(1,3)$ \\
Minas Gerais (MG) & $15(6,4)$ \\
Paraná (PR) & $1(0,4)$ \\
Paraíba (PB) & $1(0,4)$ \\
Mato Grosso do Sul (MS) & $1(0,4)$ \\
Maranhão (MA) & $1(0,4)$ \\
\hline Áreas de atuação & $\mathrm{N}(\%)$ \\
Ciências sociais aplicadas & $77(32,9)$ \\
Ciências humanas & $72(30,8)$ \\
Engenharias & $49(20,9)$ \\
Ciências exatas e da Terra & $47(20,1)$ \\
Ciências da saúde & $29(12,4)$ \\
Multidisciplinar & $27(11,5)$ \\
Ciências biológicas & $20(8,5)$ \\
Linguística, letras e artes & $17(7,3)$ \\
Ciências agrárias & $15(6,4)$ \\
\hline Fonte: Elaborado pelos autores &
\end{tabular}

Em relação ao contexto da pandemia de COVID-19, 94\% dos docentes tiveram as suas atividades transferidas totalmente para o ensino remoto e os outros $6 \%$ seguiram o modelo híbrido de ensino. Este dado é semelhante ao de outros países que transferiram o ensino em sua totalidade para a modalidade remoto, como é o caso do Marrocos e do Paraguai, e totalmente em contrapartida com os dados da Nicarágua, país caribenho que não teve alteração nenhuma na modalidade de ensino (Unesco, 2021).

Um número expressivo de 181 (77,4\%) docentes apresentaram dificuldades no ensino de uma maneira geral. Ao especificá-las, estas se resumem em: falta de capacitação ou de recursos tecnológicos por parte das instituições; problemas com o uso de tecnologias digitais (ferramentas e softwares); influência de dificuldades psicológicas; dificuldades associadas à burocracia administrativa ou com segurança de dados. Este resultado está de acordo com estudos semelhantes na literatura, que mostram que a transição do presencial para o remoto acarretou diversas dificuldades (Paludo, 2020). Como é o caso de Borba et al. (2020) que estudaram problemas durante esta transição em uma amostra de 187 professores do ensino fundamental ao pré-vestibular; e apenas um professor $(0,5 \%)$ relatou ausência total de quaisquer dificuldades durante esta transição.

\subsection{Recursos tecnológicos e capacitação ofertados pelas instituições}

A falta de recursos tecnológicos foi apontada como um dos motivos que levaram à dificuldade da atuação no ERE. Dos 181 docentes que afirmaram apresentarem dificuldades, $66,3 \%$ mencionaram algumas dificuldades, sendo que $23,8 \%$ tiveram fornecimento tecnológico insuficiente e 18,7\% não receberam nenhum recurso da 
instituição. Dos respondentes que relataram muitas dificuldades, correspondentes a $26 \%$ da amostra, 38,5\% destes tiveram fornecimento tecnológico insuficiente e 15,4\% não receberam nenhum recurso. Apenas $23 \%$ dos docentes não apresentaram nenhuma dificuldade no ERE, e destes, 83\% tiveram preparação (curso, treinamento e orientação) suficiente. Outros fatores também mencionados incluem, problemas com o funcionamento de equipamentos (exemplo: computadores, notebook) relatado por $34,6 \%$ dos docentes, falhas na conexão com a internet $(58,1 \%)$, dificuldades no uso de tecnologias digitais (31,6\%) e os aspectos burocráticos da instituição (20,5\%).

O fornecimento tanto de equipamentos tecnológicos quanto de treinamentos específicos foi testado quanto a associações às dificuldades no ERE. Mais especificamente, foi explorado o nível de dificuldades daqueles professores que receberam da instituição de ensino equipamentos, palestras, cursos, e recursos tecnológicos. Identificamos dificuldades quase três vezes menores no ensino quando os professores recebem equipamento ou dispositivo eletrônico ( $p=0,0372$; OR $=3,349)$, e quando esses têm acesso a cursos e treinamentos para o ERE ( $p=0,0094$; OR =3,176).

Os dados supracitados evidenciam a importância da preparação para o ensino ERE assim como do fornecimento de recursos tecnológicos. A dificuldade de ensinar a distância associada à falta de um treinamento ou à falta de equipamentos tecnológicos são fatores de sumária importância, principalmente porque o sucesso da transição no ensino remoto depende de fatores que incluem comunicação, tecnologia, pedagogia e comunidade (Moore et al., 2021; Jelińska \& Paradowski, 2021). Resultados que confirmam esses fatores já foram demonstrados na literatura, como por exemplo, Moore et al. (2021), que avaliou as experiências de estudantes durante a transição presencial-ERE em um campus médico na Universidade do Arkansas (University of Arkansas), e identificou que $64,5 \%$ dos alunos entrevistados tiveram uma transição satisfatória associada ao fornecimento de tecnologia e treinamento dos docentes.

\subsection{Dificuldades no uso de tecnologias digitais: ferramentas e softwares utilizados durante o ensino remoto}

Nos momentos de interação entre docentes e estudantes diferentes TDIC's foram utilizadas, como as ferramentas da Microsoft (Skype, Teams, OneDrive e o Office), do Google (Meet, Drive, Classroom, Forms, Jamboards, Docs e o Youtube), Facebook (Messenger, Facebook, Whatsapp, Instagram), plataforma Moodle, AVA, Prezi, Zoom, jogos (Kahoot, Socrative, Mentimeter), bibliotecas virtuais, Telegram e Twitter (Gráfico 1).

Entre as metodologias e ferramentas utilizadas para as aulas online, 218 docentes (93\%) fizeram uso de atividades síncronas que permitiam a interação "ao vivo" entre docente e alunos por meio de audioconferência, videoconferência ou bate-papo na web, e 210 docentes (90\%) optaram pela utilização do Google Meet para esses momentos síncronos. As ferramentas utilizadas nas modalidades síncronas, são apontadas como ideais e apresentam boa aceitação pelos estudantes, o que reforça o potencial significativo e promissor no futuro da educação (Khalil et al., 2020). Em relação a comunicação de forma assíncrona com os alunos, o WhatsApp foi utilizado por 148 docentes (63\%). A comunicação entre alunos e docentes, seja ela de maneira 
síncrona ou assíncrona, é parte essencial para o sucesso do aprendizado dos alunos no ensino remoto, principalmente durante a transição abrupta sofrida neste período (Moore et al., 2021).

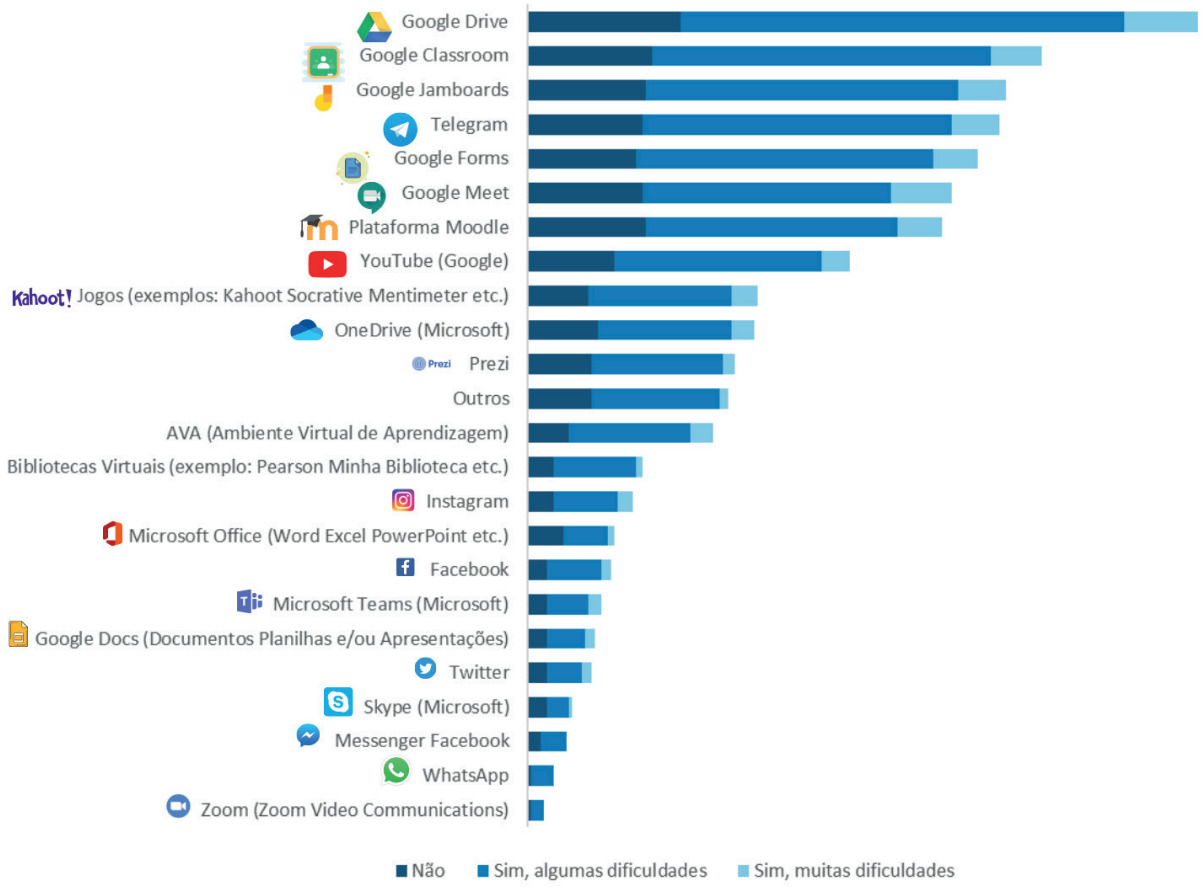

Gráfico 1. Frequência da utilização e níveis de dificuldade de acordo com cada software/ aplicativo durante o ERE.

Fonte: Elaborado pelos autores.

Apesar de que na pandemia, essas ferramentas foram frequentemente utilizadas entre os professores, e receberem ampla divulgação, 130 (55\%) docentes relataram dificuldades nas interações com os estudantes. Apenas 12\% dos docentes relataram possuir muita interação com seus alunos durante o ensino remoto. Mesmo antes da pandemia, Santaella (2013), ao abordar a utilização de tecnologias para processos pedagógicos, demonstrou que esses recursos, embora tenham o intuito de quebrar barreiras, traz à tona uma dificuldade de comunicação inesperada, relatada por 65,3\% dos professores participantes do seu estudo. Essa pouca interação pode levar a uma lacuna de comunicação entre os alunos e os docentes, e criar empecilhos que impactam na qualidade da aprendizagem online (Bacich et al., 2015; Khalil et al., 2020).

Em contrapartida, Santaella (2013) aponta que a interação entre docentes e estudantes pode ser ampliada ao explorar melhor os recursos disponíveis pelas ferramentas, tornando assim uma vantagem para a atuação em ensino remoto. Este ponto foi evidenciado no presente estudo quanto ao uso do Google Jamboards, que se trata de uma lousa interativa. Ele se destaca perante aos outros softwares avaliados nessa pesquisa, pois permitiu o dobro da interação com os alunos em comparação às outras ferramentas ( $p=0,0189 ; \mathrm{OR}=2,181)$. De maneira complementar, ressalta-se 
que os professores que usam programas do pacote Microsoft Office tendem a ter 2,6 vezes menos dificuldades no ensino remoto ( $p=0,0260$; OR $=2,687$ ), e quase duas vezes menos dificuldades ao utilizar a plataforma Moodle ( $p=0,0189$; OR $=2,181$ ).

Ao analisar o fato de ter ou não experiências ou contato anteriores com as ferramentas utilizadas no ERE e como estas refletiam em dificuldades enfrentadas pelos professores, foram identificadas diferenças estatisticamente significativas. Compararam-se professores com diferentes níveis de dificuldades: aqueles que não tiveram dificuldade alguma, aqueles que tiveram poucas, e aqueles que tiveram muitas dificuldades. A comparação mais significativa se deu no grupo daqueles que "não tiveram dificuldades" com os que tiveram "muitas dificuldades", professores que possuíam experiências anteriores com tais ferramentas tendem a apresentar quatorze vezes menos dificuldades nas atividades remotas ( $p=1,00 \cdot 10^{-6}$; OR $=14,091$ ).

Antes da pandemia, o ensino a distância era uma opção para que educadores utilizassem diferentes estratégias de aprendizagem, porém com a pandemia essa modalidade foi o único caminho para a continuidade da educação (Ismaili, 2021). Tal abordagem exigiu um grande esforço por parte do corpo docente, principalmente em relação à necessidade de elevar os níveis de competências e proficiências no uso de diferentes tecnologias (Mareketal., 2021;Al-Balas etal., 2020). Por outro lado, docentes que já possuíam experiência em EaD ou já faziam o uso de ferramentas e softwares voltados ao ensino, tiveram menos dificuldade no ERE, visto que tais tecnologias já estavam integradas em suas metodologias de ensino e cotidiano (Marek et al., 2021).

Outra pesquisa que correlacionou à experiência anterior dos educadores no ensino a distância com a percepção do enfrentamento dos alunos, demonstrou que esses docentes estimavam que seus discentes tinham menos dificuldades em comparação com professores que não tinham experiência anterior com a realização de cursos online (Jelińska \& Paradowski, 2021). Assim, os resultados encontrados aqui, demonstram que as experiências anteriores de EaD tornam a transição do ensino remoto emergencial mais positiva, quando comparado com a ausência de experiência (Marek et al., 2021).

Portanto, uma possibilidade para lidar melhor com situações semelhantes no futuro, seria a incorporação da experiência de aprendizagem por tecnologia remota e atividades online na agenda educacional regular. Assim, professores e alunos podem desenvolver importantes competências digitais, letramento digital, e se preparar para lidar melhor em outro possível evento emergencial (Shamir-Inbal \& Blau, 2021).

Outro tópico investigado por esse estudo envolveu a dificuldade dos professores em relação à sua experiência frente ao tempo de atuação como docente. Foi identificado que professores com mais tempo de profissão tendem a ter até três vezes mais dificuldades no ERE, fato evidenciado na comparação de profissionais com menos de 5 anos de profissão com aqueles que atuam entre 16 a 20 anos no ensino ( $p=0,0395$; $\mathrm{OR}=3,122$ ). Tal achado pode estar relacionado com a faixa etária desses docentes, onde os que possuem menos tempo de atuação profissional são indivíduos mais novos e podem ter melhor domínio sobre as tecnologias digitais ( $p=0,0004998)$. Este fenômeno 'tecnofóbico', medo das novas tecnologias da informação e comunicação, associado à idade já foi descrito na literatura mesmo antes da pandemia (Machado et al., 2019; Santos, 2020). Profissionais da educação mais velhos tendem a ser 
mais resistentes culturalmente às mudanças, somado a falta de preparação com tais recursos digitais. Esses profissionais podem internalizar situações de inferioridade perante aos seus colegas docentes mais novos e aos alunos que estão imersos na tecnologia e universo digital desde o nascimento (Machado et al., 2019; Santos, 2020).

\subsection{Dificuldades psicológicas e atuação do docente no ERE}

Ao associar as dificuldades psicológicas com a dificuldade de ensino, 42,7\% dos participantes apontaram implicações na saúde mental e, dentre esses, 18\% apresentaram muitas dificuldades no ensino, enquanto $73 \%$ demonstraram ter algumas dificuldades. Na análise de como problemas psicológicos externos podem refletir em dificuldades de ensino, identificou-se que os professores com algum tipo de problema psicológico tendem a apresentar quase cinco vezes mais dificuldades no ERE ( $p=$ $1,40 \cdot 10^{-6}, \mathrm{OR}=4,912$ ).

Uma parcela da amostra, 36\% não possuía uma experiência prévia com atividades em ambientes de ensino e aprendizagem virtuais. Estes aspectos psicológicos podem ter relação com as dificuldades, de uso e manuseio, junto a falta de instrução e preparo para a implementação do ERE (Araújo et al., 2020). Houve baixa interação desses docentes com dificuldades psicológicas com os alunos, sendo que apenas $9 \%$ tiveram muita interação. Além disso, diferentes falhas e problemas técnicos já foram apontadas como sendo um desafio que pode gerar dificuldades psicológicas no docente (Khalil et al., 2020).

A pandemia impactou na saúde mental de curto e longo prazo de muitas pessoas ao redor do mundo, principalmente com o transtorno de estresse pós-traumático (Maqsood et al., 2021; Usher et al., 2021). As medidas extremas de quarentena e bloqueio aumentam a sensação de solidão entre as pessoas. Esses fatores podem impactar na redução da eficácia do ensino e aprendizagem (Usher et al., 2021). Os desafios educacionais encontrados pelos docentes são mais difíceis de lidar no ensino remoto emergencial que em condições ditas "normais", ao exigir do docente preparo para lidar também com alunos que apresentam quadros de estresses e ansiedades na adaptação no ERE (Gelles et al., 2020; Usher et al., 2021).

Ademais, os novos desafios em relação ao uso das tecnologias e metodologias de ensino por parte dos docentes, também contribuíram ainda mais para sobrecarga do trabalho a distância (home office) no ERE. Este índice de trabalho excessivo para manter a produtividade acadêmica, também é responsável pelo aumento dos níveis estresse ao propiciar sintomas de ansiedade, depressão e angústia, vista aos rumos incertos decorrentes da pandemia, à busca por aquisição de conhecimento e à intensificação do fluxo de informação (Araújo et al., 2020).

É preocupante que docentes também apresentem dificuldades psicológicas sem a devida atenção durante o ensino remoto emergencial, portanto deveria existir por parte das instituições uma oferta de atendimento em saúde mental a estes profissionais. Estes problemas podem gerar bloqueios e implicações sérias impactando na performance dos mesmos durante o processo de ensino. Assim, os achados do presente trabalho corroboram com dados já apresentados na literatura, onde os desafios na saúde mental incluindo frustração, estresse e depressão não estão restritos aos alunos (Chaturvedi et al., 2021; Assunção-Luiz et al., 2021). 


\subsection{Aspectos burocráticos, avaliações pedagógicas e segurança de dados}

Entre os docentes que relataram que tiveram "muitas dificuldades"(11\%), diante da transição para o ensino remoto no contexto de pandemia; $73 \%$ apontaram dificuldades em relação a aspectos burocráticos da instituição necessários na prática pedagógica no ERE. Além disso, 69,2\% tiveram dificuldades relacionadas às avaliações dos estudantes. De todos os modelos de regressão desenvolvidos, foram identificadas duas associações significativas com a idade. Constatou-se que pessoas com idade mais avançada, tendem a ter menos dificuldades de avaliar os estudantes ( $p=0,0090, \mathrm{OR}=0,7098)$, assim como menos problemas de segurança de dados $(p=0,0005, \mathrm{OR}=0,6053)$.

Estes resultados evidenciam a possibilidade que conforme os profissionais avançam em idade, acumulam experiências de ensino e tempo de atuação. Dessa forma, mesmo em uma situação de ensino à distância emergencial, pontos vitais como avaliação dos estudantes, não foi prejudicada. Em relação a ter menos problemas com segurança de dados, estes resultados podem ser reflexo de uma postura mais conservadora apresentada por pessoas de maiores idades em ambientes virtuais.

A avaliação é indispensável para que os alunos acompanhem os resultados de aprendizagem. Existem diversas plataformas online que oferecem diferentes metodologias de avaliação, no entanto, todas elas possuem vantagens e limitações. Um ponto importante é que as instituições devem minimizar contratempos burocráticos e incentivar questões relacionadas ao ambiente educacional diante de uma pandemia (Maqsood et al., 2021). Assim, é importante lembrar que o modelo de aprendizagem online e os resultados da aprendizagem devem ser regularmente avaliados para monitoramento da sua eficácia (Khalil et al., 2020). Por outro lado, é relevante reforçar que a rápida expansão da COVID-19 não possibilitou que governos e instituições tivessem tempo hábil para um preparo adequado no regime do ERE (Daniel, 2020).

Outras dificuldades foram apresentadas como, por exemplo, o relato de $46,2 \%$ dos docentes na elaboração e realização das atividades práticas. Esses aspectos se relacionam com dados da literatura, onde se destaca que professores e gestores educacionais precisam se readaptar ao contexto do ERE a fim de manter a qualidade do ensino e da aprendizagem (Lima et al., 2020). No entanto, tais mudanças podem provocar tensões nos processos pedagógicos, ocasionando, portanto, algumas dificuldades que podem ser suprimidas a partir de novos olhares para a formação docente na atuação mediada por tecnologias (Garbin \& Oliveira, 2021).

\subsection{Lacuna e limitações do estudo}

A presente pesquisa foi realizada de forma virtual através do uso de ferramentas e aplicativos de comunicação. Ao utilizar esses tipos de abordagens os estudos estão expostos a algumas limitações, que podem interferir e/ou dificultar o andamento da pesquisa. Podemos citar a dificuldade pessoal que cada pessoa tem em utilizar alguns tipos de tecnologias, o que consequentemente pode dificultar o preenchimento do instrumento de coleta de dados utilizado neste estudo. Além disso, deve considerarse que em alguns lugares o uso de internet é mais escasso, o que também pode ter delimitado a caracterização do grupo amostral apresentado. 
Na época em que foi realizada a coleta de dados, no final de 2020, o país passava por um momento complicado em decorrência do grande aumento no número de casos de Covid-19. Dessa forma, os dados apresentados por essa pesquisa não devem ser considerados como uma realidade para ocasiões posteriores a esta data. Muitas coisas evoluíram e foram adaptadas, por isso, os autores do presente estudo encorajam para que novas pesquisas sejam feitas nesse âmbito.

\subsection{Contribuições futuras do ERE para a educação brasileira}

As adaptações e modificações realizadas durante o ERE, fizeram com que não só os docentes, mas também os alunos, ganhassem mais experiência com tecnologias digitais (TDIC's) e se apropriassem do uso de ferramentas e softwares antes não utilizados. Ressalta-se a real importância do acesso à internet para todos e computadores para todas as instituições de ensino (Universidades, institutos e escolas). Esses aprendizados poderão contribuir no futuro para uma otimização de tempo e possibilitar uma maior diversificação nas práticas utilizadas em salas de aula e nos tipos de atividades avaliativas.

Caso o ensino híbrido seja mantido, tais ferramentas poderão possibilitar que os alunos acessem conteúdos extras, diretamente de suas residências, que poderão contribuir para um melhor ensino e aprendizagem (Bacich et al., 2015). Pode-se também refletir, que atividades como as do ensino híbrido podem vir a contribuir para uma maior adesão de alunos no ensino básico, por exemplo na Educação de Jovens e Adultos (EJA), onde as pessoas passam a maior parte do dia trabalhando (Silva et al., 2021).

\section{Considerações finais}

O presente estudo permitiu identificar uma grande parcela de docentes que enfrentaram dificuldades na transição para o ensino remoto emergencial, $74 \%$ desta pesquisa, sendo elas relacionadas principalmente ao processo de migração para o ambiente virtual, uso de novas tecnologias e dificuldades psicológicas. Tais desafios podem impactar negativamente tanto na atuação dos docentes, quanto na aprendizagem dos alunos.

A familiaridade com as TDIC's é essencial para atingir o objetivo pedagógico no ensino online, e por isso, mapear e identificar os desafios técnicos é de grande importância. Trabalhos como este são relevantes pois compartilham resultados significativos que reforçam a necessidade de que pesquisadores, administradores e educadores compreendam e avaliem os processos implementados durante a transição para o ERE. Assim, essa pesquisa enfatiza a importância da incorporação de TDIC's na agenda educacional regular, o que pode contribuir para que caso surja outro evento emergencial, docentes e alunos possam estar mais preparados.

Frente aos achados do presente estudo, os autores recomendam que as IES ofereçam recursos para o acolhimento e resolutividade das dificuldades docentes, tanto quanto eventos que propiciem a promoção da saúde mental dos professores. Além disso, enfatiza-se que qualquer transição necessita de planejamento e investimento financeiro na formação adequada dos professores, para que a educação em contexto virtual alcance o mesmo desempenho observado nos momentos de aprendizagem dos encontros presenciais. 


\section{Agradecimentos}

Os autores agradecem a Coordenação de Aperfeiçoamento de Pessoal de Nível Superior (CAPES, Código Financeiro 001) e a Universidade Virtual do Estado de São Paulo (UNIVESP).

\section{Referências}

Al-Balas, M., Al-Balas, H. I., Jaber, H. M., Obeidat, K., Al-Balas, H., Aborajooh, E. A., ... \& Al-Balas, B. (2020). Distance learning in clinical medical education amid COVID-19 pandemic in Jordan: Current situation, challenges, and perspectives. BMC Medical Education, 20(1), 1-7. https://doi.org/10.1186/s12909-020-02257-4

Araujo, R. M. de., Cibelle, A., Martins, V. F., Eliseo, M. A. \& Silveira, I. F. (2020). COVID-19, Mudanças em Práticas Educacionais e a Percepção de Estresse por Docentes do Ensino Superior no Brasil. Revista Brasileira de Informática na Educação, 28, 864-891. http://dx.doi. org/10.5753/rbie.2020.28.0.864

Arroio, A., Rodrigues Filho, U. P. e da Silva, A. B. F. (2006). A formação do pós-graduando em química para a docência em nível superior. Química Nova, 29(6), 1387-1392. https://doi.org/10.1590/ $\underline{\mathrm{S} 0100-40422006000600040}$

Arruda, E. P. (2020). Educação remota emergencial: elementos para políticas públicas na educação brasileira em tempos de Covid-19. Em Rede - Revista de Educação a Distância, 7(1), 257-275. Recuperado de https://bit.ly/3voR2k1

Assunção-Luiz, A. V. A., Pitta, N. C., Cintra, A. S., ... \& Fernandes, A. P. (2021). Impacto da Covid-19 em alunos de pós-graduação. Revista Olhares \& Trilhas, 23(2), 538-554. https://doi. org/10.14393/OT2021v23.n.2.60117

Bacich, L., Neto, A. T. e De Mello, F.(2015). Ensino híbrido: Personalização e Tecnologia na Educação $\left(1^{\circ}\right.$ ed.). Porto Alegre: Penso.

Besser, A., Flett, G. L. e Zeigler-Hill, V. (2020). Adaptability to a sudden transition to online learning during the COVID-19 pandemic: Understanding the challenges for students. Scholarship of Teaching and Learning in Psychology, 1-21. https://doi.org/10.1037/stl0000198

Brasil (2020). Portaria $n^{0}$ 343, de 17 de março de 2020. Dispõe sobre a substituição das aulas presenciais por aulas em meios digitais enquanto durar a situação de pandemia do Novo Coronavírus - COVID-19. Diário Oficial da República Federativa do Brasil. Ministério da Educação.

Brasil (2019). Define as Diretrizes Curriculares Nacionais para a Formação Inicial de Professores para a Educação Básica e institui a Base Nacional Comum para a Formação Inicial de Professores da Educação Básica (BNC-Formação). Conselho Nacional de Educação (CNE). Disponível em https://bit.ly/36eEGCB.

Brasil (2016). Resolução n.510, de 07 de abril de 2016. Dispõe sobre as especificidades éticas da pesquisa nas Ciências Humanas e Sociais e de outras que utilizam metodologias próprias dessas áreas. Ministério da Saúde. Disponível em https://conselho.saude.gov. br/resolucoes/2016/Reso510.pdf

Borba, R. C., Teixeira, P. P., Fernandes, K. D. O. B., Bertagna, M., Valença, C. R. e de Souza, L. H. P. (2020). Percepções docentes e práticas de ensino de ciências e biologia na pandemia: uma investigação da Regional 2 da SBEnBio. Revista de Ensino de Biologia da SBEnBio, 13(1), 153-171. https://doi.org/10.46667/renbio.v13i1.337

Brooks, S. K., Webster, R. K., Smith, L. E., Woodland, L., Wessely, S., ... \& Rubin, G. J. (2020). The psychological impact of quarantine and how to reduce it: rapid review of the evidence. The Lancet, 395, 912-920. https://doi.org/10.1016/S0140-6736(20)30460-8 
Carneiro, L., Prata, D. N., Moreira, P. L. e Barbosa, G. V. (2019). Collaborative Learning in the Military Police of Tocantins: perspective without frontier. International Journal of Advanced Engineering Research and Science, 6(7). https://doi.org/10.22161/ijaers.676

Chaturvedi, K., Vishwakarma, D. K. e Singh, N. (2021). COVID-19 and its impact on education, social life and mental health of students: A survey. Children and Youth Services Review, 121, 105866. https://doi.org/10.1016/j.childyouth.2020.105866

Costa, E. G. e Nebel, L. (2019). O quanto vale a dor? Estudo sobre a saúde mental de estudantes de pós-graduação no Brasil. Polis, 17(50), 207-227. http://dx.doi.org/10.4067/S071865682018000200207

Daniel, S. J. (2020). Education and the COVID-19 pandemic. Prospects, 49(1-2), 91-96. https://doi. org/10.1007/s11125-020-09464-3

Dwivedi, Y. K., Hughes, D. L., Coombs, C., Constantiou, I., Duan, Y., Edwards, J. S., ... \& Upadhyay, N. (2020). Impact of COVID-19 pandemic on information management research and practice: Transforming education, work and life. International Journal of Information Management, 55, 102211. https://doi.org/10.1016/j.jijinfomgt.2020.102211

Garbin, M. e Oliveira, E. T. (2021). Por uma Nova Formação Docente: Por Que É Importante Aprender a Usar Tecnologias no Processo Formativo? Revista Cientifica de Educação a Distância, 11(2).

Gelles, L. A., Lord, S. M., Hoople, G. D., Chen, D. A. e Mejia, J. A. (2020). Compassionate flexibility and self-discipline: Student adaptation to emergency remote teaching in an integrated engineering energy course during covid-19. Education Sciences, 10(11), 304. https://doi. org/10.3390/educsci10110304

Khalil, R., Mansour, A. E., Fadda, W. A., Almisnid, K., Aldamegh, M., Al-Nafeesah, A., ... \& Al-Wutayd, O. (2020). The sudden transition to synchronized online learning during the COVID-19 pandemic in Saudi Arabia: A qualitative study exploring medical students' perspectives. BMC Medical Education, 20(1), 285. https://doi.org/10.1186/s12909-020-02208-z

Ismaili, Y. (2021). Evaluation of students' attitude toward distance learning during the pandemic (Covid-19): a case study of ELTE university. On the Horizon, 29(1), 17-30. https://doi. org/10.1108/OTH-09-2020-0032

Jelińska, M. e Paradowski, M. B. (2021). Teachers' Perception of Student Coping With Emergency Remote Instruction During the COVID-19 Pandemic: The Relative Impact of Educator Demographics and Professional Adaptation and Adjustment. Frontiers in Psychology, 12, 648443. https://doi.org/10.3389/fpsyg.2021.648443

Lima, M. C., Azevedo, S. D. e Nascimento, A. L. R. (2020). Currículo e práticas docentes durante a pandemia de 2020. Itinerarius Reflectionis, 16(1), 01-20. https://doi.org/10.5216/rir. $\underline{\mathrm{v} 16 \mathrm{i} 1.65753}$

Machado, A., Leite, C. M. F. A. e Monteiro, A. M. R. (2019). As tecnologias digitais na literatura académica da educação de adultos. LaPlage em Revista, 5(2), 86-102.

Maphalala, M. C. e Adigun, O. T. (2021). Academics' Experience of Implementing E-Learning in a South African Higher Education Institution. International Journal of Higher Education, 10(1), 1-13. https://doi.org/10.5430/ijhe.v10n1p1

Maqsood, A., Abbas, J., Rehman, G. e Mubeen, R. (2021). The paradigm shift for educational system continuance in the advent of COVID-19 pandemic: Mental health challenges and reflections. Current Research in Behavioral Sciences, 2, 100011. https://doi.org/10.1016/j. crbeha.2020.100011

Marek, M. W., Chew, C. S. e Wu, W. V. (2021). Teacher Experiences in Converting Classes to Distance Learning in the COVID-19 Pandemic. International Journal of Distance Education Technologies, 19(1). https://doi.org/1010.4018/IJDET.20210101.0a3

Moore, R., Purvis, R., Bogulski, C., Maddox, T., Haggard-Duff, L., Schulz, T., ... \& McElfish, P. (2021). Learning During COVID-19: Rapid E-Learning Transition at a Regional Medical School Campus. Journal of Regional Medical Campuses, 4(2). https://doi.org/10.24926/jrmc. v4i2.3645 
Paludo, E. F. (2020). Os desafios da docência em tempos de pandemia. Tese, 17(2), 44-53. https:// doi.org/10.5007/1806-5023.2020v17n2p44

Polydoro, S. (Coord.) (2020). Fórum de Pró-Reitores de Graduação - Boas Práticas de Ensino Remoto em 2020. https://www.youtube.com/watch?v=Rm9pxb6dZ00\&t=37s

Roggero, R. (2020). Valorização profissional e condições de trabalho docente no plano nacional de educação 2014-2024. EccoS - Rev Cient, 40, 113-28. https://doi.org/10.5585/eccos. $\underline{\text { n40.6345 }}$

Sailer, M., Schultz-Pernice, F. e Fischer, F. (2021). Contextual facilitators for learning activities involving technology in higher education: The Cb-model. Computers in Human Behavior, 121. https:// doi.org/10.1016/j.chb.2021.106794

Santaella, L. (2013). Desafios da ubiquidade para a educação. Revista Ensino Superior Unicamp, 9, 19-28.

Santos, H. M. R. (2020). Os desafios de educar através da Zoom em contexto de pandemia: investigando as experiências e perspetivas dos docentes portugueses. Práxis Educativa (Brasil), 15, 1-17. https://doi.org/10.5212/PraxEduc.v.15.15805.091

Shamir-Inbal, T. e Blau, I. (2021). Facilitating Emergency Remote K-12 Teaching in ComputingEnhanced Virtual Learning Environments During COVID-19 Pandemic - Blessing or Curse?. Journal of Educational Computing Research, 59(7),1243-1271. https://doi. org/10.1177/0735633121992781

Silus, A., Fonseca, A. L. C. e Jesus, D. L. N. (2020). Desafios do ensino superior brasileiro em tempos de pandemia da COVID-19: repensando a prática docente. Liinc em Revista, 16(2), e5336-e5336. https://doi.org/10.18617/liinc.v16i2.5336

Silva, C. R., Freitas, A. C. S. e Almeida, N. R. O. (2021). A EJA e o ensino remoto emergencial: um olhar discente. Ensino Em Perspectivas, 2(4), 1-10.

Suguimoto, H. H., Rolim, A. T., Mazzafera, B. L. e Moura, F. A. A. F. D. (2017). Avaliação do letramento digital de alunos ingressantes do ensino superior: uma abordagem exploratória do conhecimento computacional, comunicacional e informacional. Revista Brasileira de Estudos Pedagógicos, 98(250), 805-822. https://doi.org/10.24109/2176-6681.rbep.98i250.3011

UNESCO (2021). Educação: da interrupção à recuperação. Disponível em https://bit.ly/3rW6vs0

Usher, M., Hershkovitz, A. \& Forkosh-Baruch, A. (2021). From data to actions: Instructors' decision making based on learners' data in online emergency remote teaching. British Journal of Educational Technology, 52, 1338-1356. https://doi.org/10.1111/bjet.13108

WHO (2020a). Strategic preparedness and response plan. World Health Organization. Disponível em https://bit.ly/3rWutTQ.

WHO (2020b). Regional Office for Europe. Survey tool and guidance: rapid, simple, flexible behavioural insights on COVID-19. World Health Organization. Disponível em https://bit.ly/3LCfaYi.

Zhou, P., Yang, X. L., Wang, X. G., Hu, B., Zhang, L., Zhang, W., ... \& Shi, Z. L. (2020). A pneumonia outbreak associated with a new coronavirus of probable bat origin. Nature, 579(7798), 270-273. https://doi.org/10.1038/s41586-020-2012-7

\section{Como citar em APA:}

Santos, J. T. T., Assunção-Luiz, A. V., Pereira, A. L. E., Luciano, A. R. M. B., Carvalho, I. F. P. C., ... \& Garbin, M. C. (2022). Dificuldades enfrentadas por docentes do ensino superior frente ao contexto da pandemia de COVID-19. Revista Iberoamericana de Educación, 88(1), 111-126. https://doi.org/10.35362/rie8814819 